\title{
CONSTRUCTION AND THE ROLE OF SCHEMATISM IN KANT'S PHILOSOPHY OF MATHEMATICS
}

\author{
A.T. WINTERBOURNE*
}

\section{I}

THE IDEA that kantianism in the philosophy of mathematics has ceased to be tenable because of the very existence of non-Euclidean geometries is still, I think, widely held, even though the defendents of this view have recently lost some ground. It is no doubt true that the development of hyperbolic and elliptic geometries compels some reassessment of Kant's claims about the science of real space, though even here, the rigidity of kantianism can be exaggerated. It is now much more generally understood that Kant's position not only explicitly allows alternative logical possibilities, but implicitly demands their existence (1). The distinction that Kant draws is between merely logical possibility and "constructibility", where the latter term is understood in relation to pure intuition. Kant is usually interpreted as meaning that constructions in the a priori intuition of space are indispensable for geometrical science. Since both analytic and non-Euclidean geometries dispense with spatial figures (although in the latter case they may be employed'as 'analogies') Kant's theory seems far too restrictive to do further service.

The emphasis that Kant apparently places on actual spatial figures must seem somewhat naive, especially since the Cartesian programme of analytic geometry can hardly have escaped Kant's attention. How does the existence of algebraic geometry affect the orthodox - and obsolete - view that Kant is supposed to hold, viz. that spatial figures are indispensable? Of course, from a purely historical point of view there is no explanation required. Descartes' fundamental idea was to employ geometric intuition to elucidate algebraic relationships. But how did Kant understand the epistemological relationship

* City of Birmingham Polytechnic, Margaret Street, Birmingham, B3 3BX. Stud. Hist. Phil. Sci., Vol. 12, No. 1, pp. 33-46, 1981. Printed in Great Britain. 
between analytic and 'synthetic' geometry? He may have resisted the idea that the qualitative essence of figures could be reduced to numerical, and hence algebraic representations. He might have argued that analytic geometry can be seen as an analytical representation of the quantitative determinations of figures but does not capture the essence of such figures quâ spatial entities. This would accord with that general view of Kant's theory as insisting that Euclidean geometry is a description of our spatial intuition.

An alternative way of taking the relationship between Kant's theory on the one side, and analyticgeometry on the other, is provided by the Transcendental Doctrine of Method, where he makes some of his most interesting comments on the idea of construction. Kant here distinguishes mathematical and philosophical reasoning by saying that where as the latter proceeds by reasoning from concepts, the former proceeds 'synthetically', and finds its classic formulation in the axiomatic method of Euclid: the subject is founded on geometrical notions independent of algebra, and theorems are deduced from axioms by logical reasoning. That is, philosophy is reasoning from concepts; mathematics is reasoning from the construction of concepts. Algebraic geometry, on the other hand, proceeds analytically. Since Kant was convinced that his discovery of the distinction of methodology in mathematics and philosophy was of major importance, the Euclidean synthetic method must have seemed the perfect exemplification of that fact. Kant thus emphasizes the synthetic geometrical method, neglecting the analytical method for his purpose, that is, for the purpose of founding metaphysics as a science which would lead to the same certain results as geometry.

A third possibility is that Kant regarded algebra as more fundamental than either arithmetic or geometry; Cartesian geometry simply realises this idea in a way that Kant's theory can accommodate. Does Kant have any theory of algebra that might support such a reading? There is no easy answer, given that an emphasis on spatial figures pervades much of Kant's discussion in this field. Of course, since the context of much of this discussion - the Transcendental Aesthetic - is explicitly concerned with space, it may be argued that it would have been inappropriate for Kant to consider the reduction of spatial relationships to algebraic relationships. Spatial figures, in Kant's theory, still apply to the space of perception, and it is this descriptive quality of geometry with which Kant is concerned in the Aesthetic. This would suggest a much less rigid interpretation of Kantianism in the philosophy of mathematics than has sometimes been given.

It is now well understood that Kant's theory asserts the logical possibility of alternative geometries. The idea of construction is, for Kant, a constraint upon what might be called 'real' geometries, i.e. on interpreted systems that purport to apply to the space of experience. In this paper I shall emphasize one way of understanding the idea of construction in the critical writings, and link this with the doctrine of schematism. I shall then suggest that such a link provides a basis for a theory of algebra which Kant could have accepted (2). 
That Kant's theory allows for non-Euclidean geometries is an interpretation which rests on two considerations, one direct, the other indirect. The indirect consideration is that since Kant insists on the synthetic character of the propositions of geometry, the replacement of the axiom of parallels by its contrary would generate no inconsistencies in the system as a whole. Since this is indeed the case, Kant must be correct in asserting the non-analyticity of the axioms and postulates of Euclidean geometry. I have stated this argument baldly since I wish to concentrate on the direct argument that Kant employs, and I shall leave the indirect argument without further comment. Nothing I say in the sequel depends crucially on this first consideration being accepted as it stands (3). The second consideration rests principally on the following passage of the Critique:

...whence shall we derive the character of the possibility of a n object which is thought through a synthetic a priori concept, if not from the synthesis which constitutes the form of the empirical knowledge of objects? It is indeed a necessary logical condition that a concept of the possible must not contain any contradiction; but this is not by any means sufficient to determine the objective reality of the concept, that is, the possibility of such an object as is thought through the concept. Thus there is no contradiction in the concept of a figure which is enclosed within two straight lines, since the concepts of two straight lines and of their coming together contain no negation of a figure. The impossibility arises not from the concept in itself, but in connection with its construction in space, that is, from the conditions of space and its determination. (4)

Here Kant identifies mathematical 'existence' with the possibility of construction. A mathematical object - here a geometrical figure - 'exists' insofar as it can be constructed in pure intuition. Generally, Kant is taken to mean that space is given to us as being definitely and irrevocably Euclidean: what this really amounts to is the assertion that perceptual space - the space of any and every possible experience - could not be 're-constructed' such that non-Euclidean geometry provided the formal basis for intuitive, i.e. particular, constructions in space from which synthetic judgements, valid a priori, could follow.

What is involved in this idea of construction? The synthetic propositions of geometry are 'objectified' and thereby verified by constructing the 'object' of the concept in pure intuition, that is, by 'exhibiting' a priori the intuition which corresponds to the concept. The test of a 'real' geometry is this appeal to the possibility of constructing its figures - its objects - in pure intuition; more generally, the test is the possibility of intuitive construction. This means exhibiting particulars which manifest features that are true of a whole class of entities. Pure constructions in space (and time) are symbolic instantiations. Hintikka has argued that a proper understanding of Kant's philosophy of mathematics depends upon recognising that there are two distinct but related notions of 'intuition' in Kant. The mature theory links it with sensibility directly, and this meaning tends to be confused with the more restricted, and original meaning of the term, found in the pre-critical writings and the Discipline of 
Pure Reason. Here, 'intuitive' means that which represents an individual, and is contrasted with general concepts (5). It is not the spatial character of 'intuitive' constructions that is of crucial importance, but the fact that they can be employed as exemplars for a general class. The constructed figure, a triangle for instance, is the spatial representation of the 'abstract' relations which constitute 'triangularity'. The figure is useful to us precisely because it embodies those relations which are less easily grasped independently of it.

The a priori exhibition of a concept by means of an intuitive construction may consist in a simple empirical procedure such as making marks on paper or moving the beads of an abacus. A natural interpretation of Kant's meaning of construction - or 'exhibiting in intuition'-is by means of the logical procedure of existential instantiation. The construction is a 'particular' which is effectively the concept made flesh: thus construction is a general way of allowing the deduction of $\mathrm{F}(\mathrm{a})$ from the existentially quantified sentence $(\exists \mathrm{x})(\mathrm{Fx})$. The test of the meaningfulness of a concept - its 'real' rather than 'merely logical' possibility-is the construction of a figure. This is produced a priori-in a way that is somewhat analogous to syllogistic reasoning, which is determination of particular conclusions under general rules by means of the faculty of judgement - yet is at the same time 'an appearance present to the senses'(6). This idea-that Kant's philosophy of mathematics can be 'reconstructed' by appeal to quantification theory - is one of the main points of Hintikka's interpretation. The use of the natural deduction rule of existential instantiation introduces new representatives of individuals - and this, on Hintikka's view, is what Kant's use of intuitive construction involves, and pre-dates the Aesthetic's use of 'intuition' where it relates to spatial intuition directly. According to Hintikka, the idea that the mathematical method is based on the use of general concepts in concreto-that is, in the form of individual instances - provides the starting-point for Kant's mature theory of mathematical reasoning (7). Kant's view may be identified in a very general way with his claim against rationalist metaphysics that 'existence' is not a predicate:

...all existential propositions are synthetic... Anything we please can be made to serve as a logical predicate; the subject can even be predicated of itself; for logic abstracts from all content. But a determining predicate is a predicate which is added to the concept of the subject and enlarges it. (8)

We shall see later that for Kant the function of schemata is to 'particularise' certain concepts, that is, to present in intuition individuals which represent a general class.

It should not be assumed that Kant thinks of this figurative construction as complete in itself; this would reduce the process of construction to an empirical procedure, valid for the presented spatial figure, but limited to it. This would of course fail to yield the characteristics bound up, for Kant, with the recognition of mathematical truth, viz neceissity and universality. For instance, in order to obtain synthetic propositions about triangles it is not sufficient merely to consider the concept 'triangle:' such a procedure yields only analytic propositions. However, if we exhibit the triangle in intuition, i.e. if we draw a triangle, or think of one in imagination, then such a construction putatively generates the body of synthetic propositions, valid a priori, with which Euclidean geometry has made us 
familiar (9). Clearly, this shows again that there must be something more in the notion of construction than merely the production of lines on paper or images in imagination. And Kant does indeed supply the required feature. So that the constructed figure may be 'adequate to the concept' Kant goes on to explain the procedure in terms of transcendental imagination, i.e. in terms of a priori conditions. There must be an element in the procedure that is 'presuppositional': in this way, the empirical construction is given an a priori 'kick.' We now ask again: how can we be certain that what can be 'read off' the individual figure is valid for all possible figures of this kind? Kant's answer is that in employing the imagination to construct a triangle in pure intuition we uncover-by 'regressive analysis' - the a priori conditions by which imagination is itself bound in producing particular figures of this kind.

The single figure which we draw is empirical, and yet it serves to express the concept, without impairing its universality. For in this empirical intuition we consider only the act whereby we construct the concept, and abstract from the many determinations... which are quite indifferent, as not altering the concept 'triangle .'(10)

This consideration of an act, presupposed in the empirical construction, supplies the necessary presuppositional element. (I shall return to this below.) Mathematics, Kant insists, does not extend knowledge by analysis of concepts alone: verification in mathematics requires that it 'hasten to intuition'. In pure intuition the concept is instantiated and considered in concreto, yet non-empirically, since the construction is in pure,"not empirical intuition. The concept is 'particularised,' i.e. constructed, and whatever follows from the universal conditions of the construction, is universally valid of the object of the concept thus constructed. In order to produce a particular construction which is adequate to the concept, we require some form of mediation between understanding - the faculty of rules which at the same time provides a priori concepts - and sensibility, in whose domain the constructions must be presented if they are to acquire existential significance, i.e. sense (11). In this way, we effect an isomorphism between the $a$ priori truths which belong to the concept 'triangle' and the identifiable a priori conditions exemplified in the construction. In Kant 's hierarchy of faculties, it is judgement that has the task of subsuming under rules, and is in general the procedure of moving from a major and a minor premiss of a syllogism to a particular conclusion. In this way Kant introduces - as part of the Transcendental Doctrine of Judgement - the idea of schemata of pure concepts of understanding:

If understanding in general is to be viewed as the faculty of rules, judgement will be the faculty of subsuming under rules. (12)

It is the notoriously difficult Schematism chapter which expands the implications of mathematical construction and contributes to a less constricted understanding of Kant's philosophy of mathematics.

We have seen that the figure produced in intuition from which synthetic propositions, valid a priori, may be 'read off,' must in some matter be representative of all figures of that kind (13). Any characteristic possessed uniquely by the 'empirical' figure can be abstracted 
and ignored in the reasoning process. How can a single figure perform such a task adequately? As Kant admits, no image could be adequate to the general concept 'triangle'(14). The answer lies in the notion of the transcendental schematism.

\section{III}

At this point it will be helpful to rehearse some of the keyideas relating to 'synthesis' in the critical philosophy, as preparation for the use made of this concept in the chapter on the schematism itself. The idea of construction is much wider and of more general significance in Kant than focussing on his philosophy of mathematics may suggest. It is initially located inside such a framework, but is generalised as the process of 'synthesis of the empirical manifold'(15). This synthesis, as I suggested above, provides the presuppositional or transcendental element by means of 'imagination.' The connection between concepts and intuitions is effected by means of a synthesis of which the schematism is the focal example.

Synthesis in general... is the mere result of the power of imagination, a blind but indispensable function of the soul, without which we should have no knowledge whatsoever, but of which we are scarcely ever conscious. To bring this synthesis to concepts is a function which belongs to the understanding, and it is through this function of the understanding that we first obtain knowledge properly so-called.(16)

This is the mirror-image of the specific case of mathematical construction. In mathematical construction we procude, by means of an 'imaginative synthesis,' an image for a concept:

The image is a product of the empirical faculty of reproductive imagination: the schema of sensible concepts, such as figures in space, is a product and, as it were, a monogram, of pure a priori imagination, through which, and in accordance with which, images themselves first become possible.(17)

To subsume particulars under concepts is the task of the faculty of judgement in general, and the schematism in particular. The productive synthesis of imagination is a transcendental act:

We cannot think of a line without drawing it in thought, or a circle without describing it... Even time itself we cannot represent save insofar as we attend, in the drawing of a straight line (which has to serve as the outer figurative representation of time) merely to the act of the synthesis of the manif old whereby we successively determine inner sense, and in so doing attend to the succession of this determination in inner sense.(18)

Connection - synthesis of the manifold - is not a merely passive process undertaken by sensibility and intuition, but is an active procedure of the faculty of imagination. Time, as formal intuition, demands synthesis of imagination quâ transcendental act: as form of intuition, time is the undifferentiated phenomenon of lapse, and yields only the possibility 
of determinate succession (19). The successive synthesis of the manifold - an act performed by means of the productive imagination-locates this whole problem for Kant within transcendental philosophy' (20). Geometry itself - 'the mathematics of space [Ausdehnung]' - is grounded in the productive imagination in the generation of figures. It is on this basis that axioms are understood as conditions of a priori intuition in figurative construction.

In the transcendental deduction Kant had argued that the pure concepts of understanding apply to objects of intuition in general. However, such concepts are for this reason incapable of giving determinate knowledge of objects:

The pure concepts of understanding relate... to objects of intuition in general... through which no determinate object is known. (21)

It is schemata that 'particularise' concepts in the required sense. Only the schematism, quâ transcendental act, can provide determinate knowledge of objects.

\section{IV}

The schema is a product of imagination. It is a universal procedure - an act - which provides an image for a concept.

It is a rule of synthesis of the imagination, in respect to pure figures in space. (22)

The schema of a 'sensibilised' concept - in this case a spatial figure - is a product of pure a priori imagination, through which, and in accordance with which, images-something empirical-first become possible. It is not, 'transcendentally' speaking, the constructed triangle as such which is the ground of a priori valid synthetic propositions, but rather the fact that it has been produced in accordance with the schema for 'triangle,' either as a figure on paper or imaginatively. Images are connected with the concept by means of the schema which they designate (23). This schema for 'triangle' is a rule of procedure for construction in intuition. Without such an a priori rule of construction we could not be certain that we had in fact produced a triangle. The drawing is a particular which 'presents' an instance of the class 'triangle,' and thus represents this class. This enables us to recognise the figure quâ instance of a geometrical class rather than, say, an undifferentiated spatial area, or any of the various other possibilities which are implicit in the empirical construction (24). As Kant points out, in mathematics we consider the universal in the particular,

...or even in the single instance, though still always a priori and by means of reason. Accordingly, just as this single object is determined by certain universal conditions of construction, so the object of the concept, to which the single object corresponds merely as its schema, must likewise be thought as universally determined. (25)

We may object to this idea on the ground that it is, in an important sense, superfluous. Kant needs an 'image' - a spatial figure produced in intuition - in order that a priori valid synthetic propositions may be 'read off. Yet might it not be said that the empirical 
construction serves as an heuristic aid rather than a necessary component of the reasoning process? The schema, as a rule of procedure for constructing any image for a concept, must 'contain,' abstractly, or 'pre-constructively,' all of the 'information' that can in principle be included in, and thus 'read off' from, the intuitive construction quâ particular instantiation. If this were not the case, the constructed figure could not be 'adequate to the concept' - that is, there would be either more or less 'information' in the empirical figure than is in the concept. This 'rule' of construction should contain, in principle, all that the geometer requires in order to 'reason' about triangles. The body of such rules would be a geometry without figures. More precisely, it would provide a 'geometry' which dispenses with spatial constructions.

The idea of such an 'act' of imagination can only be understood in the context of the notion of synthesis to which I alluded above. Nonetheless, evenf or Kant it seems miraculous how such functions of the imagination can be the foundation for a system of relations which, when spatially interpreted, generates an a priori science which has application to experience, whilst yet nothing much can be said of it except that it exists. In talking of the schematism, Kant is at one point reduced to admitting that it is

...an art concealed in the depths of the human soul, whose real modes of activity nature is hardly likely ever to allow us to discover, and to have open to our gaze. (26)

Nevertheless, the conception of the schematism implies that geometrical science could dispense with spatial constructions. However, it could not dispense with 'temporal' constructions, since time - as the form of inner sense - is the necessary condition of all experience, outer - that is, spatial - and inner - that is, minimally temporal and maximally spatio-temporal. It is synthesis of the manifold of pure a priori intuition which gives knowledge of objects. This synthesis, or 'taking up and connecting,' is the result of the transcendental procedures of imagination, and as a function effecting the subsumption of intuitions under general concepts is the task of transcendental schematism:

...if this manif old is to be known, the spontaneity of our thought requires it to be gone through in a certain way, taken up and connected. This act I name synthesis. (27)

This is best understood in relation to Kant's 'definition' of number. To think a number 'in general' is the representation of a method

...whereby a multiplicity... may be represented in an image in conformity with a certain concept. (28)

Number is, in Kant's cryptic formulation,

...simply the unity of the synthesis of the manifold of a homogeneous intuition in general. (29)

The 'movement' of consciousness produces undifferentiated succession in the manifold if inner sense: 'synthesizing' the manifold is 'taking up' and 'connecting.' Number in general is the presented product of such synthesis (30). Crudely, a number is simply a conventional 
way of marking a determinate position in the manifold of inner sense: numbers are a 'sensuous epistemological tool' (31). It should be recalled that schemata are not themselves spatial images: they are a priori determinations of time in accordance with rules, which make images possible (32). This locates Kant's 'pure science of time' within transcendental philosophy. Time is more general - less dispensable - than space: the 'science of time' must therefore be more fundamental than geometry quâ science of space. The pure science of time is not arithmetic, since this has actual numbers as its objects and is insufficiently general. The science of 'number in general' which, through its connection with the fundamental transcendental synthesis of the manifold of inner sense concerns 'taking up' and 'connecting' in an arbitrary way, and is therefore the condition for the possibility of both arithmetic and geometry, is algebra.

Mathematics does not only construct magnitudes (quanta) as in geometry; it also constructs magnitudes as such (quantitas), as in algebra. In this it abstracts completely from the properties of the object that is to be thought in terms of such a concept of magnitude. ... Once it has adopted a notation for the general concept of magnitudes so far as their different relations are concerned, it exhibits in intuition, in accordance with certain universal rules, all the various operations through which the magnitudes are produced and modified. Thus in algebra, by means of a symbolic construction, just as in geometry by means of an ostensive construction, we succeed in arriving at results which discursive knowledge could never have reached by means of mere concepts.(33)

In his theory of geometry Kant appears to insist on the indispensability of figures in space. Yet the teaching of the schematism, centering as it does on the fundamentally temporal nature of rules of synthesis for generating figures in space, links algebra to the intrinsically temporal character of construction by means of the symbol. Kant's theory indirectly suggests that spatial constructions are dispensable, provided that we are in possession of an adequate system of symbols by means of which any intuitive, that is, particular relations, can be expressed. The algebraic method is not 'geometrical,' but it is constructive in the required sense, that is, it employs variables the only acceptable value of which are individuals (34). The concepts expressed through and instantiated in the symbols - especially those concerning relations of magnitude - are presented in intuition: they are symbolically instantiated. The sine quâ non of geometrical science for Kant is not the existence of spatial figures, but the construction in pure intuition, i.e. the possibility of considering the universal in the particular construction (35). This may be either a spatially extended figure, or an algebraic representation of the relations expressed in such a figure. In Kant's letter to Schulz he says that 'universal arithmetic', i.e. algebra, is an ampliative science and that the remaining parts of pure mathematics (mathesis), progress largely because of algebra, considered as the universal theory of quantities. As Hintikka has pointed out, Kant's theory of mathematical reasoning, and especially the interpretation of intuition which emphasises its non-spatial character, can be identified in the so-called pre-critical writings. As early as 1763 , Kant had distinguished mathematical from 
metaphysical reasoning by means of the former's use of signs, known 'individually and sensibly,' which give concrete knowledge of general concepts. (36)

This interpretation of construction and schematism seems to me to be consistent with the explicit remarks on algebra to be found in the Critique of Pure Reason. However, a serious problem of exegesis would appear to be raised by some typically convoluted remarks made in the Critique of Judgement, which bear on this issue, and suggest a fundamental inconsistency in Kant's use of terms. In section 59 of the Critique of Judgement Kant draws some distinctions between schemata and symbols whith cannot easily be reconciled with his more detailed comments on the use of mathematical notation made elsewhere. Kant there says that all concepts demand 'verification' by means of intuitions. This is part of what is meant by Kant's assertion that thoughts without content are empty, and intuitions without concepts are blind. Neither concepts without a corresponding intuition, nor intuition without concepts can yield knowledge (37). Empirical concepts are verified by 'examples,' pure concepts by schemata. This process of verification, or 'rendering in terms of sense' can take place in either of two modes:

Either it is schematic, as where the intuition corresponding to a concept comprehended by the understanding is given a priori, or else it is symbolic, as where the concept is one which only reason can think, and to which no sensible intuition can be adequate. In the latter case the concept is supplied with an intuition such that the procedure of judgement in dealing with it is merely analogous to that which it observes in schematism. In other words, what agrees with the concept is merely the rule of this procedure, not the intuition itself. (38)

So far there is no difficulty: where the concept is an idea of reason such that that there is, in principle, no intuition that could be adequate to it, the expression of the concept is made by means of a symbol. (An 'Idea of Reason' is a concept that is neither abstracted from, nor applicable to sense-experience: it 'transcends the possibility of experience'(39).) The relationship between a symbol and its concept is merely analogous to the manner in which a schema relates to its concept. The schematic and the symbolic are both, for Kant, intuitive modes of representation: the difference is that the former directly 'present' the concept through demonstration, while the latter are merely indirect 'presentations' of the concept by means of analogy. This interpretation of symbolism is what one would expect, given the critical philosophy's insistence on the transcendent character of certain concepts of reason. It is clear that such concepts could only be given intuitive-and hence immanent - meaning through analogies of some kind. However, Kant thereupon identifies both schematism and symbolism as 'hypotyposes,' i.e. presentations (Darstellungen, exhibitiones) and not mere marks, (Charakterismen). Marks are

...merely designations of concepts by the aid of accompanying sensible signs devoid of any intrinsic connection with the intuition of the object. Their sole function is to afford a means of reinvoking the concepts according to the imagination's law of association-a 
purely subjective role. Such marks are either words or visible (algebraic or even mimetic) signs, simply as expresssions for concepts. (40)

This presents a serious problem: here Kant is identifying algebraic symbols as merely conventional marks, whose purpose is to reinvoke concepts by means of simple association. before, I suggested that algebraic expressions directly presented in intuition relations of magnitude as such, so that they could be connected to the rules of synthesis described as schematism. But here, Kant appears to place algebraic notation inside his wider concept of symbolism, rather than inside a wider concept of schematism. The relationship between an algebraic symbol and a number concept should be direct, and is quite different from the relationship that a model or analogy has to that concept of reason for which it is a model or analogy. The connection between a symbol quâ analogy and its concept is looser than the connection between schemata and their concepts. One thing may be. used as a symbol for another by virtue of the similarity in the 'structure of reflection' in the two cases:

In this way a monarchical state is represented as a living body when it is governed by constitutional laws, but as a mere machine (like a hand-mill) when it is governed by an individual absolute will; but in both cases the representation is merely symbolic. For there is certainly no likeness a despotic state and a handmill, whereas there surely is between the rules of reflection upon both and their causality... In language we have many such indirect presentations modelled up on an analogy enabling the expression in question to contain, not the proper schema for the concept, but merely a symbol for reflection. (41)

Thus symbols quâ analogies may express concepts for which the direct employment of an 'intuition' is out of the question. In the Critique of Judgement the idea of representation by means of analogy is usded as Kant's central designation of symbolism, and although this idea is itself clear enough, algebraic symbolism should not be found within its compass.

An explanation for this confusion may be the Critique of Judgement's concern with so-called reflective judgement, in contrast to a determinant judgement. If the 'universal' - in the shape of a rule, principle or law-is given, the judgement which subsumes the particular under it is determinant: if, on the other hand, only the particular is given, then reflective judgement concerns finding a universal for it. Determinant judgement - the subsumption of particulars under rules - operates

...even where such a judgement is transcendental and, as such, provides the conditions a priori in conformity with which alone subsumption under that universal can be effected. (42)

Thus unlike transcendent judgements - where ideas of reason can only be represented by means of analogies - transcendental judgements - where a priori conditions of knowledge are involved - can be made determinate bymeans of schemata. Nowsince rules of synthesis of a priori imagination are presupposed in all construction of mathematical objects, such objects must present their concepts, and make them determinate, in a way that is quite different from reflective judgement by means of 'symbols,' which, as Kant 
says, is representation by 'mere analogy.' Kant's general theory of mathematical construction mitigates against any consideration of algebraic notation as mere marks, even though such a notation is 'conventional.' These symbols are important as practical devices, even though the a priori locus of mathematical construction is the procedure of imaginative synthesis (43).

\section{NOTES}

1 - For instance, see Gordon Brittan, Kant's Theory of Science (Princeton University Press, 1978), Chap. 3, p. 68.

2-Cf.C. D.Broad, Kant:An Introduction (Cambridge: Cambridge University Press, 1978), p. 69.

3-Cf. G. Brittan, op. cit., pp. $43 \mathrm{ff}$ and 68.

4-Kant, Critique of Pure Reason, trans. by N. Kemp. Smith (Macmillan, 1973), B268.

5-Hintikka's various interpretations of Kant's philosophy of mathematics emphasize the 'synthetic' character of the idea of 'exhibiting in intuition,' and relate this notion in Kant to Euclid's Elements. In what follows, my debt to Hintikka will be obvious, and I believe that my understanding of the theory of algebra to be found in Kant dovetails with Hintikka's general position. See J. Hintikka, 'Kant on the Mathematical Method,' The Monist 51 (1967), 352-375; 'Kant's "New Method of Thought" and Theory of Mathematics,' Ajatus 27 (1965), 37-47; 'Kant's Notion of Intuition,' The First Critique, T. Penelhum and J.H. MacIntosh (eds) (Belmont, 1969), pp. 38-53.

6-A240/B299.

7-J. Hintikka, 'Kant on the mathematical method,' The Monist 51 (1967), 359.

$8-\mathrm{A} 598 / \mathrm{B} 626$.

9-A716/B744. If this is established, Kant believes he has justified his theory of space as $a$ priori intuition. The transcendental exposition has the task of showing that only on the assumption of this characteristic view of space can the possibility of geometry as a body of synthetic propositions, valid a priori, be understood.

10-A714/B742, my emphasis. 
$11-\mathrm{A} 240 / \mathrm{B} 299$.

$12-\mathrm{A} 132-133 / \mathrm{B} 171-172$.

13 - A property unique to figures in Euclidean space. In non-Euclidean 'spaces' of variable curvature-Lobatschefskian, for instance - it may not be possible to construct similar triangles of different magnitudes. In homogeneous Euclidean space this is always possible.

14 - This problem clearly reminds us of Berkeley's struggle against 'abstract general ideas'. Cf. Gerd Buchdahl, Metaphysics and the Philosophy of Science (Blackwell, 1969) p. 285: "When a geometer appears to "reason round a triangle on a blackboard," he is not drawing general conclusions from a particular triangle; but neither is he "really thinking" of some 'abstract universal triangle'. Rather, he is reasoning about those properties of his triangle which are held in common with the class of triangles concerned in the demonstration, that is to say, with all those triangles which are given the properties mentioned in the definitions, postulates and axioms'.

15-G. Buchdahl, op. cit., p. 556, especially note I.

16-A78/B103, Kemp Smith's emphasis: the language here reminds us of the schematism: cf. A141/B181.

$17-\mathrm{A} 141 / \mathrm{B} 181$.

18-B154-155, my emphasis.

19-G. Buchdahl, op. cit., p. 642.

20-B155 and note; also A163/B204.

$21-\mathrm{B} 150$.

22-A141/B180.

23-A142/B181; I have modified Kemp Smith's translation here. 
24-The active, interpretative process of 'seeing an aspect' requires imagination in a Kantian sense. Wittgenstein confirms Kant's insight when he concludes that the concept of 'seeing an aspect is akin to the concept of (forming) an image'. Philosophical Investigations (Oxford, 1953) p. 213. The general idea can also be found in E. Cassirer, The Philosophy of Symbolic Forms, Vol. 3 (Yale University Press, 1957) p. 200.

25-A714/B742, my èmphasis.

26-A141/B181; cf. also B103.

27-A77/B102, my emphasis.

$28-\mathrm{A} 140 / \mathrm{B} 179$.

$29-\mathrm{A} 143 / \mathrm{B} 182$.

$30-\mathrm{A} 103$.

31 - Kant, Selected Pre-critical Writings, trans. by G. Kerferd and D. Walford (Manchester University Press, 1968), p. 24: 'Enquiry concerning the Clarity of the Principles of Natural Theology and Ethics', 1763.

$32-\mathrm{A} 145 / \mathrm{B} 185$.

33-A717/B745, my emphasis.

34-Hintikka, 'Kant on the mathematical method' etc., p. 359. Cf. also Kant, A159-160/B198-199.

35-Cf. note 14, and Brittan, op. cit., pp. 53 ff.

36-Kant, 'Enquiry etc.', pp. 13 and 24; also Philosophical Correspondence, ed. and trans. by A. Zweig (University of Chicago Press, 1967), p. 129.

$37-\mathrm{A} 51 / \mathrm{B} 75$. 
38 - Critique of Judgement, trans. by James Creed Meredith (Oxford, 1957), Section 59, p. 221.

39-A320/B377.

40 - Critique of Judgement, loc. cit., my emphasis.

41-Critique of Judgement, p. 223.

42-Critique of Judgement, p. 18.

43 -Cf. A. Heyting, Constructivity in Mathematics, Proceedings of Colloquium (Amsterdam, 1957; North Holland 1959), p. 70. 